\title{
E-Generaciones: ¿Cuánto hay de Adultocéntrico en el Análisis de la Relación entre la Población Infantil y las Nuevas Tecnologías?
}

\section{E-Generations: How Adult-centric is the Analysis of the Children and New Technologies Relationship?}

\author{
Iván Rodríguez Pascual \\ Universidad de Huelva
}

\begin{abstract}
Resumen. La irrupción de las Nuevas Tecnologías de la Información y la Comunicación (TIC) ha propiciado un debate sobre sus consecuencias en la vida de los niños y niñas que habitan la sociedad de la información. El propósito de este texto es analizar críticamente los diagnósticos parciales sobre la cuestión. Partiendo de la conjetura de que éstos vienen a estar anclados en una visión que subestima las capacidades del niño/a como agente social, detectamos una perspectiva adultocéntrica que infravalora el papel de estas generaciones socializadas en el uso de los nuevos medios electrónicos en tanto que usuarios competentes. Frente a esta posición defendemos, apoyándonos en evidencias empíricas, que la población infantil puede jugar un papel más activo y crítico que redunde en un uso positivo de las mismas.
\end{abstract}

Palabras clave: nuevas tecnologías, infancia, sociología, cambio social.

\begin{abstract}
The coming of New Technologies (ICTs) is leading us to a discussion about their consequences in children's life in the context of the information society. The aim of this paper is to analyze from a critical approach the main diagnosis about this topic. The starting idea is that they are characterized by an image of non-capable or immature children as technology user. It is argued in this article that some empirical evidences exist pointing out the fact that e-generations can play a more active and central role in the new technologies use.
\end{abstract}

Keywords: new technologies, childhood, sociology, social change.

\section{Introducción}

Aunque los niños han tardado un poco más en incorporarse a ella masivamente, la infancia entró muy pronto a formar parte de las cábalas y futuribles que inauguraron la llegada de la Sociedad de la Información. Puede parecer una paradoja, pero no lo

La correspondencia sobre este artículo debe enviarse al autor a la Escuela Universitaria de Trabajo Social, Departamento de Sociología y Trabajo Social, Campus del Carmen, Avda. Fuerzas Armadas s/n, 21071 Huelva, España. E-mail: ivan@uhu.es. es si se entiende el valor expresivo que la infancia tiene como icono y como representación en el seno del discurso adulto. Paidólatras o no, lo cierto es que entender la infancia como un campo de proyecciones cruzadas que hacen referencia a las preocupaciones y prioridades del mundo adulto que la construye como categoría estructural de cada sociedad, eso que a veces se llama la sentimentalización de la infancia (Jenks, 1996), nos permite abordar hechos singulares relacionados con los cambios sociales impulsados por las nuevas tecnologías. Por ejemplo, 
que cuando Internet sólo era una quimera para buena parte de los niños y niñas del planeta, e incluso un raro privilegio en los hogares de las sociedades industrializadas, la revista Time decidiera en julio de 1995 utilizar la imagen de un niño asustado frente a una computadora para representar uno de los hábitos de consumo incipiente (y alarmantes) de los primeros usuarios de la red: el cibersexo y el acceso a la pornografía en formato digital (Barba y Montes, 2007).

Lo que pretendemos afirmar es que, desde sus inicios, la cuestión de la relación entre las llamadas nuevas tecnologías y la población infantil comenzó a construirse, no tanto desde el análisis de experiencias concretas y datos empíricos, como desde conjeturas que entroncaban decididamente con una representación del campo de fenómenos muy significativa y anclada a la imagen de un niño vulnerable y poco autónomo en tanto usuario tecnológico. Más una víctima de la tecnología que un usuario propiamente dicho.

Las páginas de este texto constituyen una revisión de algunas de las perspectivas y evidencias que han dominado la configuración del discurso adulto en las últimas décadas, prestando una especial atención a la necesidad de cambiar hacia un enfoque más riguroso a la hora de contemplar las capacidades de la población infantil, por un lado, y la complejidad del contexto social en el que los niños se mueven, por otro. Para ello revisamos primeros el enfoque clásico en el análisis de las consecuencias del advenimiento de la sociedad de la información en la vida infantil, a nuestro juicio claramente adultocéntrico, para después dar cuenta de los últimos hallazgos que parecen sustentar una interpretación alternativa.

\section{Planteando los problemas: de la muerte a la liberación de la infancia a través de las nuevas tecnologías}

Casi una década antes del niño de la portada de Time, Postman (1984) ya había especulado con la posibilidad de que las pantallas, y en espacial la televisiva, fueran un caballo de Troya destinado a dinamitar desde dentro la idea misma de infancia como espacio separado del de los adultos. Y aunque se indica con frecuencia que existe un contraargumento que viene a señalar en un tono ciertamente naíf, quizás cayendo en el mismo error cargado de esencialismo, que la tecnología es, más bien, el espacio natural de la infancia y la condición para su liberación del mundo adulto ${ }^{1}$, no es menos cierto que este segundo discurso tiene muy poco eco en los medios y también menos peso en el diagnóstico de las consecuencias de la irrupción dicha tecnología en la vida infantil. Lógicamente, la prevalencia de un diagnóstico armado sobre el cimiento de la representación del campo de fenómenos desde la perspectiva de la ruptura con el pasado, el riesgo potencial y la construcción, a efectos expresivos, de un usuario pasivo y vulnerable, conlleva una pre-selección de temas y objetos de investigación que acaba reforzando las conjeturas de partida.

Así, todavía antes de que despuntara el nuevo siglo, ya habíamos acumulado cierta masa crítica de publicaciones relevantes que daban cuenta de los efectos perniciosos de estas nuevas tecnologías de la información y la comunicación, la mayor parte de ellas concentradas en torno a un racimo de problemas y circunstancias que reflejan, sobre todo, la cara oculta de las aplicaciones tecnológicas. Encontramos así planteamientos referidos, por ejemplo, a:

- Acceso a contenidos impropios, perjudiciales o desagradables que pueden generar rechazo o perturbación en los niños y uso de la red para diseminar mensajes ofensivos y discriminatorios de carácter racista, machista, etc.

- Distribución e intercambio de pornografía infantil al amparo del anonimato de las salas de Chat y listas de distribución, así como otras actividades relacionadas con la paidofilia.

- Consecuencias perjudiciales debido al abuso de Internet o el uso del PC de carácter físico (obesidad, desarreglos posturales, etc.) o psicológico (depresión, aislamiento, adicción).

- Uso de la red por parte de los propios menores de edad como herramienta para el acoso y el bullying, así como para otras actividades

\footnotetext{
1 Véase, por ejemplo, el texto de Tapscott, (1998).
} 
(supuestamente) delictivas como la descarga de contenidos audiovisuales de la red (fundamentalmente software, música y películas) ${ }^{2}$.

Aparecen de esta forma algunas referencias fundamentales con una temática variada: por ejemplo los estudios de Funk y colegas sobre los efectos de los videojuegos sobre la conducta infantil, así como la preferencia (fundamentalmente masculina) por el videojuego violento (Buchman y Funk, 1996; Funk et al., 2000; Kirsh, 1998). Igualmente, no faltan las evidencias sobre la conexión entre las nuevas tecnologías y el abuso, el aislamiento o el mal ajuste familiar y escolar (Stanley, 2001; Wolack, 2003) así como sus efectos sobre el desarrollo (Subrahmanyam et al. 2000). Por último, uno de los temas que más claramente se perfilan como amenaza global, incluso al propio concepto de infancia, es la cuestión del acceso a la pornografía o, lo que es peor, la implicación de menores de edad en la producción y distribución de pornografía infantil a través de la red (Freeman-Longo, 2000; Mc Cabe, 2000).

La insistencia en los aspectos más conflictivos de las nuevas tecnologías y la proyección de una imagen empobrecida del niño como usuario tecnológico $\mathrm{y}$ agente social ha casado bien con el conjunto de los temores de padres, educadores y adultos en general. Temores que las posibilidades de estas mismas tecnologías, cada vez más potentes y aparentemente impredecibles, parecen alentar tal y como el combustible alimenta el fuego.

Sin embargo, sin despreciar la validez científica de estos hallazgos, puede objetarse que están excesivamente focalizados en áreas problemáticas de la conducta infantil (abuso, adicción, violencia, etc.) y que provienen, en su mayor parte, de diseños metodológicos experimentales o cuasiexperimentales, así como otros escenarios en los que niños y niñas son más un objeto de estudio ${ }^{3}$ que sujetos co-investigadores. Haciendo buena la afirmación de que toda

\footnotetext{
${ }^{2}$ En algunos informes sobre el uso que los menores de edad hacen de las nuevas tecnologías las actividades relacionadas con la descarga de contenidos protegidos quedan reflejadas como "delitos cometidos por menores en Internet". Nos parece que éste es un buen ejemplo de lo que queremos designar con el término "adultocentrismo": se prefiere criminalizar al menor con arreglo a un marco legal de por sí escurridizo y cuestionado por la comunidad de internautas antes que intentar comprender el sentido de la acción (véase, por ejemplo, el informe de 2002 encargado por la Comunidad de Madrid y disponible en la dirección www.protegeles.com).
}

práctica investigadora es una manifestación de pautas culturales que revela mucho sobre nuestras prenociones en torno al concepto de infancia, y que respecto a la innovación tecnológica pueden resultar reiterativas (Wartella y Jennings, 2000; Woodhead y Faulkner, 2000), no es descabellado suponer una dosis generosa de adultocentrismo entre los presupuestos que animan esta clase de investigación. Adultocentrismo identificado en: a) el planteamiento sesgado de los temas de interés, que recalcan aspectos conflictivos o problemáticos o, en su caso, de interés preferente de los adultos y de las instituciones sociales relacionadas con el control y socialización infantil, b) la preferencia por diseños metodológicos que esconden tanto la complejidad del estatus del niño como aquellos aspectos netamente contradictorios de la sociedad que les acoge y c) que deja poco espacio para que estas e-generaciones se expresen y hagan valer sus razones y las de sus pares en el análisis de sus condiciones de vida en el contexto de la sociedad de la información y las nuevas tecnologías. Por lo demás, aspectos fundamentales desde el punto de vista del propio desarrollo infantil, como lo relacionado con la construcción del tiempo y las actividades de ocio o el papel del niño como productor $-\mathrm{y}$ no sólo consumidor pasivo- de contenidos en el ciberespacio, han pasado casi desapercibidos en el conjunto de la literatura científica que explora las consecuencias de la irrupción de las nuevas tecnologías en la vida infantil (Buckhingam, 2004).

\section{Nuevos enfoques en el análisis: hacia una pers- pectiva del protagonismo infantil en el contexto de la sociedad de la información}

Una explicación plausible a la insistencia con que buena parte de la literatura especializada y el discur-

\footnotetext{
3 La acotación no es gratuita ni caprichosa: en muchas de las fuentes de información que se citan comúnmente en este campo los niños y niñas ni siquiera han intervenido como sujetos encuestados, lo cual arroja sobre la información que sopesamos la sospecha de ser distorsionada o poco precisa. Así sucede, por ejemplo, con el Eurobarómetro Especial $n^{\circ} 250$ de 2006 de la UE [Safer Internet] o, por poner un ejemplo local, con la Encuesta sobre Equipamiento y uso de Tecnologías de la Información y Comunicación en los hogares del Instituto Nacional de Estadística; instrumentos ambos en los que se seleccionan hogares en los que viven menores de 18 años pero no son éstos los entrevistados sobre sus pautas de consumo tecnológico, sino sus padres, tutores u otros parientes
} 
so público ha venido focalizando sus esfuerzos en el estudio de los usuarios más vulnerables, corresponde a una suerte de proyección de temores que pertenecen propiamente al rango de fenómenos del mundo adulto, pero que permean fácilmente nuestros diagnósticos. Como señalan Steinberg y Kincheloe (2000): "parece que el genio de la infancia tradicional está fuera de la botella y no puede volver a ella". Es decir, es un hecho que muchos adultos aprecian la diferencia entre la infancia vivida, en un entorno más estable en el que la frontera con el mundo adulto constituía, por así decirlo, la propia experiencia infantil y la infancia presente, caracterizada por absorber de manera problemática buena parte de las transformaciones sociales que han sacudido muchas instituciones fundamentales desde el último cuarto del siglo XX, y particularmente la familia y la educación. Si a esto añadimos que la propia tecnología constituye uno de los principales núcleos de temores difusos tan afines a nuestra existencia posmoderna (Bauman, 2007) es fácil comprender lo que subyace tras la célebre imagen de "la muerte de la infancia”. Así, lo que los adultos fácilmente pueden apreciar es que la falta de apoyo ins- tecnológico es un hecho difícilmente reversible. En el contexto de una infancia construida como intersección de múltiples intereses políticos, comerciales, pedagógicos etc. La única posibilidad de mantener a la población infantil completamente alejada de las consecuencias positivas y negativas de las nuevas tecnologías pasa por un acuartelamiento forzoso en los barracones de la institucionalización y la impermeabilización de los hogares frente a la diseminación de estas tecnologías. Hoy ya nadie contempla esta posibilidad: por políticamente incómo$\mathrm{da}^{4}$ pero también inviable: resulta difícil pensar en un control efectivo del acceso a las nuevas tecnologías que no recuerde al célebre dicho de "poner puertas al campo".

Así, en la práctica los hogares con niños son los mejor equipados tecnológicamente, precisamente aquellos en los que los adultos y los que no lo son tanto son los principales usuarios de las distintas aplicaciones tecnológicas de las TIC. Los datos del entorno europeo lo corroboran ${ }^{5}$ : Internet es una presencia mayoritaria en estos hogares, si bien la diferencia se reduce drásticamente en lo que se refiere al acceso al PC.

Tabla 1. Indicadores básicos sobre Tecnologías de la Información según hogares con niños (UE-15) 2007

\begin{tabular}{lccc}
\hline & & $\mathbf{2 0 0 7}$ & \\
\hline & Hogares con niños* & Hogares sin niños* & Todos los hogares \\
\hline$\%$ tienen acceso a Internet en la casa & 72 & 53 & 59 \\
$\%$ tienen acceso a un PC** & 41 & 59 & 46 \\
\hline $\begin{array}{l}\text { Fuente: Eurostat (acceso: *con/sin menores dependientes (notación de Eurostat) } \\
\text { **a través de uno de sus miembros }\end{array}$ & & \\
\hline
\end{tabular}

titucional, la conceptualización de los problemas de la infancia como problemas privados y el hecho de que la inmersión del niño en las nuevas tecnologías se hace más respondiendo a los intereses comerciales de la industria del entretenimiento que a un criterio de bondad educativa, lo han convertido en un actor dominante pero expuesto permanentemente al riesgo de ser instrumentalizado. Sin embargo, el objetivo de convertir al niño en un protagonista responsable de su propio futuro exige que empecemos a avanzar más allá.

Quizás un primer paso para reconducir los intereses de los investigadores pase por admitir que la implicación de las nuevas generaciones en el medio
En el caso español la progresión con que nuestras e-generaciones se han volcado en el uso de las nuevas tecnologías no es menos sorprendente, como puede apreciarse en el gráfico siguiente elaborado con los últimos datos proporcionados por el INE. El hecho de que una abrumadora mayoría de los niños y niñas entre 10 y 14 años ya dispongan de un telé-

\footnotetext{
${ }^{4}$ ¿Cómo hacer compatible la llamada a la nueva cuarentena infantil con el discurso glorificador sobre el advenimiento de la sociedad de la información y sus progresos?

$5 \mathrm{El}$ estado de las fuentes secundarias sigue siendo, más bien parte del problema. Todavía son muy pocos los datos que, con carácter comparativo y referidos al acceso y uso de la tecnologías por parte de las nuevas generaciones, encontramos en los servidores de Eurostat. Y son menos todavía los que tienen a los propios niños como informantes.
} 
Gráfico 1. Niños españoles de 10-14 años y uso de NT 2003-07

\section{España: Niños 10-14 años que usan NT 2003-08}

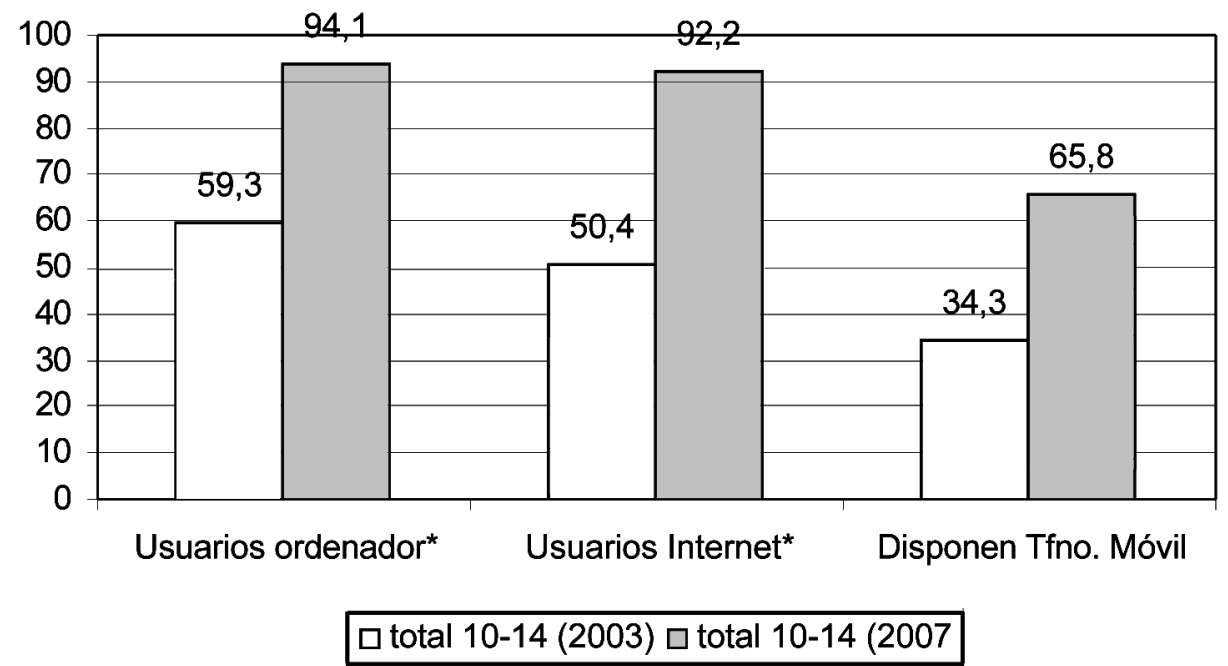

Fuente: INE, Encuesta sobre Equipamiento y Uso de Tecnologías de la Información y Comunicación en los hogares.

fono móvil de uso propio ${ }^{6}$ y sean usuarios activos de Internet coloca a la población infantil española en el disparadero tanto de los nuevos riesgos como de las potencialidades beneficiosas de las TIC, y permite introducir un concepto novedoso no exento de contornos borrosos: los estilos de vida digitales, también referidos a la cultura infantil (Feixa, 2006).

Por tanto, la única vía para superar un enfoque estrictamente adultocéntrico y acercarnos a ese difícil equilibrio que supone el objetivo de proteger sin estrangular y evitar los riesgos maximizando los beneficios que, siguiendo a Ruxton (2005), parece la única alternativa para ser respetuosos tanto con nuestras obligaciones como adultos como con los derechos de la población infantil, es la de promover una autonomía responsable que permita un papel central de niños y niñas en tanto usuarios informados y en las condiciones más seguras posibles. Esto implica tener en cuenta, desde una perspectiva cercana a los intereses del niño, tanto las potencialida-

${ }^{6}$ Un hecho más frecuente en la sociedad española que en otros lugares de la Unión Europea, algo que sólo puede explicarse aludiendo a la especificidad cultural de la sociedad menos natalista del planeta, pero que difícilmente puede derivar de la tecnología misma, que es igual en todas partes. des de las nuevas tecnologías como sus riesgos inherentes. Y ello contando con un enfoque suficientemente complejo que incluya dimensiones imbricadas tales como (Hasebrink et al, 2007): las variables socioeconómicas que explican diferentes usos y accesos a la tecnología; el papel mediador de los propios adultos y también de los grupos de pares en la construcción de actividades en línea; e igualmente variables contextuales que no suelen ser tenidas en cuenta pero pueden tener un papel determinante en la propia construcción social de la infancia como el marco legal que regula las TIC, el discurso público sobre los riesgos de Internet para la infancia o el conjunto de actitudes relativas a la infancia y la tecnología, por ejemplo.

En este sentido, en el terreno de la investigación con niños y adolescentes es un hecho reconocido de manera cada vez más consistente que hemos solido infraestimar sus capacidades. En el campo de los estudios sobre tecnología Buckingham (2002) recoge el testigo y señala: los niños son usuarios más habilidosos de lo que pensamos. Lo cual implica que lo son para escapar a los filtros de bloqueo, o a las restricciones paternas y otras impuestas por las autoridades educativas; pero también para elaborar con- 
tenidos útiles a otros menores, mostrar apoyo en sus redes de amistad o introducir a miembros de otras generaciones mayores en el uso de Internet. Con estos mimbres se fabrica una investigación sobre la infancia y las nuevas tecnologías que apuesta por la observación de las conductas infantiles en contextos naturales, el método etnográfico y la proyección del papel del niño hacia una labor más participativa dentro de los propios diseños de investigación. Lógicamente, la representación de la relación entre infancia y nuevas tecnologías desde esta perspectiva resulta más amplia y compleja.

Por ejemplo, sin eludir los aspectos problemáticos de la revolución tecnológica que representan las TIC, las nuevas generaciones electrónicas han demostrado poseer niveles de competencia relativamente altos que pueden actuar, modelados convenientemente, como factores protectores. El hecho de que hayamos tendido a subestimar las propias capacidades de los niños y adolescentes como usuarios de tecnología explica porqué hay todavía poca investigación empírica que explore concienzudamente un aspecto central de la relación entre la infancia y las nuevas tecnologías como es la de la gestión del riesgo. Esta idea es chocante para los adultos, que han convertido el riesgo en uno de sus privilegios, pero resulta insoslayable si queremos entender el papel que juegan estas e-generaciones en la sociedad de la información. Distintos autores (Lenhart y Madden, 2007; Olesen, 1999) confirman que la inmersión en las nuevas tecnologías viene acompañadas de estrategias para tratar de minimizar los riesgos que supone el nuevo entorno virtual: desplazarse desde las salas de chat a los servicios de mensajería o establecer nicks o apodos y regular el contacto dentro de las redes sociales conocidas, por ejemplo, son dos buenas muestras de lo que llamamos mecanismos de gestión del riesgo. Mecanismos necesarios toda vez que dichas aplicaciones tecnológicas han dejado de ser objetos individuales de consumo y se han convertido, merced a la propia acción transformadora de la cultura infantil, en sistemas relacionales y soportes de dicha cultura y la interacción entre pares en la que existe y se reproduce (Gil, Rivero y Gil, 2003). Incluso los casos en los que expone información privada en el entorno cibernético, difícilmente puede asumirse que sea algo acci- dental sino una actividad grupal y cultural en la que los niños escriben para sus pares en un entorno que les brinda un espacio mucho más participativo y libre que el proporcionado por la tecnología convencional o el mundo real, encorsetado en la jaula de hierro de la institucionalización (Moinian, 2006).

Teniendo en cuenta que tanto los riesgos asumidos como la competencia aumentan con la edad (Hasebrink et al, 2007), es razonable esperar que estas estrategias también se hagan más complejas conforme nos adentramos en la adolescencia y se entrevea ya la juventud. La experiencia personal del autor de estas páginas, derivada de los estudios empíricos que ha conducido ${ }^{7}$, es que muchos de los riesgos asumidos por chicos y chicas en su vida diaria, y en particular los que tienen que ver con el contacto con extraños y el acceso a información poco apropiada tenían mucho que ver con su origen social y el capital cultural de sus familias, y hubieran sido fácilmente minimizados con más asesoramiento adulto que mejorara las propias estrategias infantiles de gestión del riesgo.

Para la construcción de una nueva perspectiva destinada a entender la relación entre la infancia y la tecnología desde una óptica menos adultocéntrica y más sustentada en una visión participativa del papel de la población infantil, es igualmente útil resaltar los aspectos de esta relación en la que niños y niñas aparecen como co-constructores de la misma. Lógicamente, además de los propios niños, su partenaire son los adultos, tanto figuras del entorno familiar como otras personas de referencia, normalmente ejerciendo múltiples papeles que van más allá del mero control restrictivo o vigilancia. Holloway y Valentine (2001) por ejemplo, insisten en la importancia que la negociación y definición de las situaciones en las que la tecnología está presente adquiere en el contexto de la interacción con el mundo adulto e institucional. Aarsand (2007) Viene a profundizar aún más en esta interacción y destaca el papel que los niños juegan en el contexto de la brecha digital entre generaciones, ofreciendo un perfil bifronte en el que los mismo son sujetos orientados que agentes activos en la tarea de incorporar a sus mayores al uso de las últimas aplicaciones tecnoló-

7 Véase, por ejemplo: Rodríguez (2005). 
gicas. De otro lado, como señala Rodríguez (2005), la relación de los adultos con la aparición y diseminación de estas tecnologías está plagada de aspectos contradictorios que explican, en parte, muchas variables referidas a la infancia: valga como muestra el posicionamiento de los padres frente al uso del teléfono móvil, condenando por un lado su uso abusivo y su dependencia del mismo, pero proporcionándolo masivamente bajo el argumento de que ayuda a establecer una comunicación inmediata y contribuye a la seguridad de los hijos cuando están fuera de casa.

\section{Discusión}

En el terreno del diagnóstico de las consecuencias de la aparición de las nuevas tecnologías en la vida infantil los argumentos son muchos pero, con ser rigurosos, no todos reflejan con la misma precisión lo que los niños y niñas sienten y experimentan en sus vidas cotidianas en el contexto de la llamada sociedad de la información. Puede que haya quien entienda que esto no es importante, pero es complicado generar análisis válidos sobre la cuestión si la realidad social nunca es conocida directamente sino a través de voces silenciadas o mediadas por otros referentes cuyos intereses y vivencias pueden ser diferentes a los de los propios niños, cuando no simplemente contradictorios. Precisamente, para que las nuevas tecnologías de la información y la comunicación no sean un problema y sí una herramienta con usos potencialmente beneficiosos para la población infantil y adulta, necesitamos estos análisis válidos.

Hemos identificado un tipo de discurso caracterizado por una insistencia en aspectos problemáticos y la suposición de que buena parte del problema es el carácter inmaduro y vulnerable de la población infantil como usuaria de tecnología. Dicho discurso suele estar referido, por lo general, al área de la institucionalización de la población infantil y en particular al ámbito doméstico y escolar (controlados mayoritariamente por el adulto), y utiliza metodologías que objetivan a los menores de edad, frente a otras más participativas en las que niños y niñas puedan tener un papel cercano al de la co-investigación. Éste ha sido etiquetado como adultocéntrico por constituir un típico producto discursivo en el que el etnogrupo adulto (nosotros) tiende a proyectar una imagen preconcebida sobre los diagnósticos de la realidad social, más que a contar con el grupo de "los otros" (en este caso los niños) para elaborar un retrato de la misma que esté más cerca de la propia experiencia infantil.

Sin embargo, utilizando igualmente referencias científicas que optan por diseños más participativos, generalmente con un carácter etnográfico, en las que se exploran áreas en las que los niños tienen un papel activo y central y es inevitable referirse a la existencia de una cultura infantil, o al menos de cierta interacción grupal característica alejada de los controles directos, parece posible generar una perspectiva diferente sobre el impacto que las TIC tienen en las vidas cotidianas de niños y niñas. No sólo se han presentado datos que apuntan a que dichas tecnologías son ya un hecho en el mundo de la infancia y parte de los estilos de vida digitales, no una tenencia futura a la que podamos escapar. Además, la población infantil parece jugar un papel especialmente activo en ellas y, lejos de ser un usuario pasivo, juega su papel en la negociación de las normas y límites que los adultos fijan para esta nueva realidad. En la conformación de espacio relacional virtual que podría ser una respuesta a una institucionalización creciente de la experiencia cotidiana de los niños, pero también en lo que se refiere a la evitación del riesgo a través de mecanismos que nacen y se mantienen en el seno de este mismo espacio relacional. En este contexto, algunas de las actividades categorizadas como peligrosas o de riesgo adquieren un significado distinto como prácticas transgresoras destinadas, en muchos casos, a parodiar y/o criticar las prácticas y valores del mundo adulto.

Creemos que existen evidencias que apuntan a que esta perspectiva de análisis, más comprometida con el respeto por las singularidades del mundo infantil y la importancia de hacer participar a los niños en los diseños de investigación, lejos de trivializar sobre los riesgos que acechan en torno a las nuevas tecnologías proporciona una plataforma de análisis extremadamente útil que puede aprovechar ese papel activo de los menores de edad justamente en sentido contrario: reforzando los facto- 
res protectores que, inscritos en las propias capacidades de niños y niñas, conduzcan a una minimización de los riesgos sin merma de las muchas poten- cialidades ofrecidas por la innovación en el campo de las tecnologías de la información y la comunicación.

\section{Extended Summary}

\section{Problem}

The coming of New Technologies (ICTs) has leaded us to a discussion about their consequences in children's life in the context of the information society. Many studies are starting considering the main risks and problems of these new information and communication technologies, such as Internet or mobile phones. According to the last research, results, new media are eroding very fast the foundations of what was considered the classical childhood, especially the frontiers between adult and child life. Due to the extremely easy connection and the apparently lack of control over hazardous contents and cybernetic encounters, most of these studies remark how adult people are losing control about how much danger new technologies are producing. A special attention has been paid to the question of pornography and sexual exploitation of children in the net, constituting a double problem: children and young people who can access pornographic contents but also people who can be used to produce these pornographic contents. At the same time, a depiction of other new negative consequences over children's life is presented. It has been said, for example, that new technologies are highly addictive and cannot be controlled by minors. Technology is supposed to cause psychological effects like depression, anxiety or even loneliness, inviting children to withdraw from real world, having poor cybernetic relationships.

Taking this information as a starting point, the aim of this text is to analyse from a critical approach these kind of diagnosis about the consequences of new technologies in children's life. This work tries to give an answer to questions like:

- Are these new risks a representative description of the social world of children in the context of the information society?

- Is this analysis considering the implications of what is specifically different in children's culture?
- Are we considering sufficiently the point of view of children and young people when thinking of their lives under the sign of technological innovations?

\section{Methods and results}

This is not a text providing certain results directly from raw data, but a text that works on the analysis of the contradictions that can be found on scientific literature nowadays. Our method is rooted in the review of the different evidences and results present in this scientific publications around the topic of new technologies of information and their influence in children's lives. It is also a chance to analyse the kind of methodology that is used when children's world is watched from the point of view of adult researchers. A starting point in this analysis is that research is not a neutral work but a cultural product that reveals also our underlying conception or representation of childhood. For this reason a kind of reflexive analysis about research and researchers is also needed when we try to understand the trends of social change in children's lives in the context of information society, as well as the role of adult and younger people in these trends.

The main result of this approach is remarking how ambivalent the scientific production can be around this topic, but also how this ambivalence is rooted in two different ways of understanding not only childhood and children, even how the research about them is made. As it is described in the text, in most of the scientific production that identifies new technologies as a source of danger and negative effects in childhood, children play a little role and are more observed objects than subjects. The research using participative methods and studying children in context usually leads to evidences and discussions where risks is not the dominant topic, making easier an ambivalent interpretation of the consequences of these new technologies. The first 
kind of research has been labelled in this article as "adult centric". The second one can be identified as "naturalistic" or "contextual".

\section{Discussion}

The aim of this text is to analyse critically the diagnosis about the consequences of new technologies in children's life. As a result of the approach to different evidences and perspectives in the context of scientists work, we start with the idea that an adult centric point of view is characterized by an image of non-capable or immature children as a technology user. After considering different approaches, it seems clear that experimental designs as well as researchers that do not consider the possibility of studying children in their own social contexts and taking into consideration the idea of children's social participation could be producing a skewed representation of children's life related to technology. From a sociological point of view it can also be stated that new risks exists in the society of information, but some of them they are not exactly new and, at the same time, the dominant literature about the topic could be offering a depiction of children's life excessively focused in risks and paying little attention to positive uses.

The term adultcentrism has been used in this article to describe the trend in some kind of research to: a) choosing objects of study related to adult interests and point of view rather than those closer to children and focusing research in the topic of control, b) choosing research designs where the child is more and object of study than a subject participating in the research and c) leaving just a little space to hear the voice of the generations of digital natives that are being grown in the sometimes contradictory trends and discourse of the information society that adult people are building with them.

In spite of just believing that children and young people can be described in terms of risk and victimization, It is argued in this article that some empirical evidences exist in a naturalistic or contextual approach that point to the fact that e-generations can play a more active and central role in the matter of use of new technologies. For example, being more creative and skilled as expected, or using information technologies to gain social support or reinforcing relationships in real life. The point in this perspective is that leads to the empowerment of children as subjects of social change. In this sense, it is not our purpose to minimize or underestimate the real risks of new media, but to invite to include children from a participatory approach in the diagnosis. This approach means that participatory methodologies should be paid more attention because they help to collect children discourse to the understanding of a complex phenomenon as it is the forthcoming of information society. As part of what has been called the digital native perspective, children and their contexts can be key informants, pieces and valuable subjects in the process of research. This way the focus moves from the adult notion of risk to a definition of risk and other relevant phenomenon from a negotiated perspective where children are something more than observed objects.

\section{Referencias}

Aarsand, P.A. (2007). Computer and Video Games in Family Life: The digital divide as a resource in intergenational interactions. Childhood, 14, 235256.

Barba, A. y Montes, J. (2007). La ceremonia del porno. Barcelona: Anagrama.

Baumann, Z. (2007). Miedo líquido: la sociedad contemporánea y sus temores. Barcelona: Paidós.

Buchman, D. y Funk, J. B. (1996).Video and Computer Games in the 90's: Children's Time commitment and game preference Children Today, $24, \mathrm{n}^{\circ} 12-15$.

Buckingham, D. (2002). Crecer en la era de los medios electrónicos Madrid: Morata.

Buckingham, D. (2004). The Media Literacy of Children and Young People: A review of the research literature on behalf of Ofcom (obtenido 12-06-2007, acceso on-line: www.ofcom.org.uk).

Feixa, C. (2006). Estilos de vida de los niños en la cultura digital Panorama Social 3, 54-63.

Freeman-Longo, R. E. (2000). Children, Teens and Sex on the Internet, Sexual Addiction \& Compulsivity, 7, 75-90. 
Funk, J. B. et al. (2000). Preference for Violent Electronic Games, Self-Concept, and Gender Differences in Young Children American Journal of Orthopsychiatry, 70, 233-241.

Gil, A., Feliu, J, Rivero, I. Gil, E. P. (2003). ¿Nuevas tecnologías de la información y la comunicación o nuevas tecnologías de la relación? Niños, jóvenes y cultura digital. UOC. [obtenido: 30-102003, acceso on-line www.uoc.edu/dt/20347/ index.html].

Hasebrink, U., Livingston, S., Haddon, L., Kirwil, L. Ponte, C. (2007). EU Kids On-line: Comparing Children's Online Activities and Risk across Europe, a Preliminary Report Comparing Findings for Poland, Portugal and UK Londres: London School of Economics and Political Science (obtenido 01-12-2008, acceso on-line: www.eukidsonline.net).

Holloway, S. L. y Valentine, G. (2001). It's Only as Stupid as You Are': children and adult's negociation of ICT competence at home and at school Social and Cultural Geography, 2, 25-42.

Instituto Nacional de Estadística: Encuesta de tecnologías de la información en los hogares. Madrid: INE.

Jenks, C. (1996). Childhood Londres: Routledge.

Kirsh, S. J. (1998). Seeing the World through 'Mortal Kombat' Colored Glasses: Violent videogames and the development of a short term hostile attribution bias Childhood, 5, 177-184.

Lenhart, A. y Madden, M. (2007). Teens, Privacy \& Online Social NetworksHow teens manage their online identities and personal information in the age of MySpace (obtenido 24-06-2007, acceso on-line: www.pewinternet.org).

Mc Cabe, K. A. (2000). Child Pornography and the Internet, Social Science Computer Review, 18, 7376.

Moinian, F. (2006). The Construction of Identity on the Internet: Oops! I've left my diary open to the whole World! Childhood, 13, 49-68.

Olesen, J. (1999). Children and Media Risks, Odense: Department of Contemporary Cultural Studies (University of South Denmark).

Postman, N. (1984). The Disappearance of Childhood. Nueva York: Delacorte Press.

Rodríguez Pascual, Iván (2005). Revisando críticamente el discurso sobre el impacto de la sociedad de la información en la población infantil: el problema del aislamiento social, Papers, 77, 4579.

Ruxton, S. (2005). Children's Rights in the EU: Next Steps (EURONET).

Stanley, J. (2001). Child Abuse and the Internet, Issues in Child Abuse Prevention, $\mathrm{n}^{\circ}$ 15, (obtenido el 15/02/2002 acceso on-line: www.aifs. org.au).

Steinberg, Sh. R. y Kincheloe, J.L. (comps.) (2000). Cultura infantil y multinacionales Madrid: Morata.

Subrahmanyam, K. et al. (2000). The Impact of Home Computer Use on Children's Activities and Development, The Future of Children. Children and Computer Technology, 10, 123-144.

Tapscott, D. (1998). Growing up digital: the rise of the next generation Nueva York: McGraw Hill

Wartella, E. A. y Jennings, N. (2000). Children and Computers: New Technology-Old Concerns, The Future of Children. Children and Computer Technology, 10, 31-43.

Wolak, J. et al. (2003). Escaping or connecting? Characteristics of youth who form close online relationships, Journal of Adolescence, 26, 105119.

Woodhead, M. y Faulkner, D. (2000). Subjects, Objects or Participants? Dilemmas of Psychological Research with Children En Christensen, P. y James, A. Research with Children: Perspectives and Practices Londres: The Open University.
Manuscrito recibido: 04/01/2010

Revisión recibida: 19/01/2010

Manuscrito aceptado: 22/02/2010 